\title{
Microangiopathic Hemolytic Anemia:A Rare Clue to Diagnose Bone Marrow Metastatic Gastric Adenocarcinoma
}

\author{
Md. Shahriar Siddiki, ${ }^{1}$ Nur Alam, ${ }^{1}$ Istiuqe Ahmed,${ }^{1}$ Md. Kamrul Hasan Patwari, ${ }^{1}$ Md. Habibur Rahman, ${ }^{1}$ Rajib \\ Bhowmic, ${ }^{2}$ Minahj Uddin Bhuiyan, ${ }^{3}$ Sarmistha Biswas, ${ }^{4}$ Md. Faizul Islam Chowdhury ${ }^{5}$
}

\begin{abstract}
:
Microangiopathic Hemolytic Anemia (MAHA) is a hematological condition which is very rare for the primary presentation of a gastric adenocarcinoma with bone marrow metastases. When it emerges as initial findings in a previously undetected case of malignancy, the diagnosis is often missed and results in inappropriate management. Carcinoma stomach associated with MAHA is generally having fulminant course. This is a case report of a 30-year-old male who presented with widespread bone marrow infiltration along with Coomb's negative haemolytic anemia, thrombocytopenia and schistocytes in peripheral blood typical of MAHA. The combination of MAHA and bone marrow infiltration in gastric adenocarcinoma is a very rare entity. When the cause of progressive MAHA is unknown, the possibility of cancer-associated MAHA must be excluded by performing additional tumor workup, including the detection of tumor markers, Endoscopy of upper GIT, colonoscopy, bone marrow examinations, and PET-CT or bone scans.
\end{abstract}

Keywords: Microangiopathic hemolytic anemia (MAHA); gastric adenocarcinoma; bone marrow metastasis.

(c) (1) (2) (2)

DOI: https://doi.org//0.3329/jom.v20i2.420I2

Copyright: (C) 2019 Siddiki MS et al.This is an open access article published under the Creative Commons Attribution-NonCommercial-NoDerivatives 4.0 International License, which permits use, distribution and reproduction in any medium, provided the original work is properly cited, is not changed in any way and it is not used for commercial purposes.

Received: 02 September, 2018;

Accepted: 06 January, 2019

\section{Introduction:}

Microangiopathic hemolytic anemia (MAHA) is a rare paraneoplastic syndrome in different solid tumors specially in advanced adenocarcinoma. ${ }^{1}$ It is defined as a severe hemolytic anemia with a negative Coombs test and schistocytes in the peripheral blood smear. ${ }^{2}$ When a malignancy first presents as MAHA, it is often misdiagnosed and treated as thrombotic thrombocytopenic purpura (TTP). Progressive MAHA of unknown origin, therefore, warrants a search for occult malignancy. Among malignant conditions, association with carcinoma stomach, lung, breast, unknown

1. Indoor Medical Officer, Department of Medicine, Dhaka Medical College Hospital (DMCH)

2. Assistant Registrar, Department of Medicine, DMCH

3. Registrar. Department of Medicine, DMCH

4. Associate professor, Department of Medicine, DMCH

5. Professor, Department of Medicine, DMCH

Corresponding author: Dr. Md Shahriar Siddiki, Indoor Medical Officer, Department of Medicine, Dhaka Medical College Hospital, Dhaka.E-mail: dr.md.s.siddiki@gmail.com. primary and lymphoma are common. ${ }^{2}$ Bone marrow study in such cases may help locate the primary by unmasking tumor deposits, but extensive bone marrow necrosis (BMN) is another unusual finding in disseminated malignancy. However, both MAHA and BMN indicate grave prognosis in this setting.

\section{Case summary:}

A 30-year-old, sailor, previously normotensive, nondiabetic male presented with low grade intermittent fever without chill and rigor for 8 months with a maximum recorded temperature of $100^{\circ} \mathrm{F}$, which mostly came at evening, subsided spontaneously or by taking medication with sweating. Seven days prior to admission in hospital patient has been suffering from high grade, continued fever with a maximum recorded temperature of $104^{\circ} \mathrm{F}$ without chills and rigor and not subsided completely with medication. Just before the day of admission patient became semiconscious, couldn't recognize his own people and talked irrelevantly. There was no history of diarrhea, vomiting, urinary 
complaints, cough, Haemoptysis, contact with TB patients, hematemesis, melaena, skin rash, oral ulcer, joint pain, history of travel to malaria endemic zones, IV drug abuse, any other bleeding manifestation. Physical examination showed a GCS score of 4/15, severely anaemic, mildly icteric and dehydrated without any cyanosis, clubbing, koilonychia, Leuconychia, lymphadenopathy, thyromegaly. Pulse - 118/min, Blood pressure- $110 / 70 \mathrm{~mm}$ of $\mathrm{Hg}$, temperature- $103^{\circ} \mathrm{F}$, respiratory rate $-21 / \mathrm{min}$. Systemic examination revealed altered level of consciousness with left sided hemiplegia as evidenced by unable to localize the painful stimuli by left upper limb and no movement of left lower limb, bilateral extensor plantar response with no signs of meningeal irritation and no apparent cranial nerve palsy. Other systemic examinations were normal. On routine blood examination, his haemoglobin level was only $3.3 \mathrm{~g} / \mathrm{dL}$ with an elevated WBC count of $20,240 / \mathrm{mm}^{3}$ and a low platelet count of $14,000 / \mathrm{mm}^{3}$, ESR- 40 $\mathrm{mm}$ in $1^{\text {st }}$ hour. Initial Peripheral blood film revealed leucoerythroblastic blood picture with thrombocytopenia. Repeated $\mathrm{CBC}$ results showed same picture but subsequent PBF revealed schistocytes, anisocytosis, polychromatic RBC, few nucleated red blood cells with thrombocytopenia suggestive of MAHA. Then Work-up to identify the cause of haemolysis was initiated on an emergency basis. Liver function tests showed serum bilirubin- $1.9 \mathrm{mg} / \mathrm{dl}$, SGPT- 34 , lactate dehydrogenase (LDH) - $1184 \mathrm{U} / \mathrm{L}$. Serum creatinine was $1.5 \mathrm{mg} / \mathrm{dl}$, reticulocyte count $-8.06 \%$, FDP - $74.16 \mathrm{mg} / \mathrm{dl}$, D-dimer - $1.5 \mathrm{ug} / \mathrm{ml}$. Direct and indirect Coomb's tests were negative ruling out immune mediated hemolysis. Routine urine analysis revealed albumin+, pus cell- 10-12/HPF, RBC15-20/HPF. ANA, ICT for malaria, blood culture (Fan method), HBsAg were negative. CT scan of brain was normal. USG of Whole Abdomen revealed few prominent upper para aortic and mesenteric lymph nodes ( $3.4 \times 1.6 \mathrm{~cm} \&$ smaller), slightly coarse hepatic parenchymal echotexture with prominent spleen. The patient received supportive transfusions without significant improvement in blood counts. With TTP as diagnostic consideration and for the evaluation of total presentation a bone marrow study was done which showed small clumps of medium sized cells seems to be of nonhaemopoitic origin, some cluster exhibit glandular pattern; Comment: suggestive of secondary metastasis of bone marrow. Then upper GIT endoscopy was done and it revealed large area of diffuse nodular irregular growth seen at the body and part of the antrum (Figure 1). Biopsy result from the gastric antrum revealed poorly differentiated adenocarcinoma. (Figure 2).

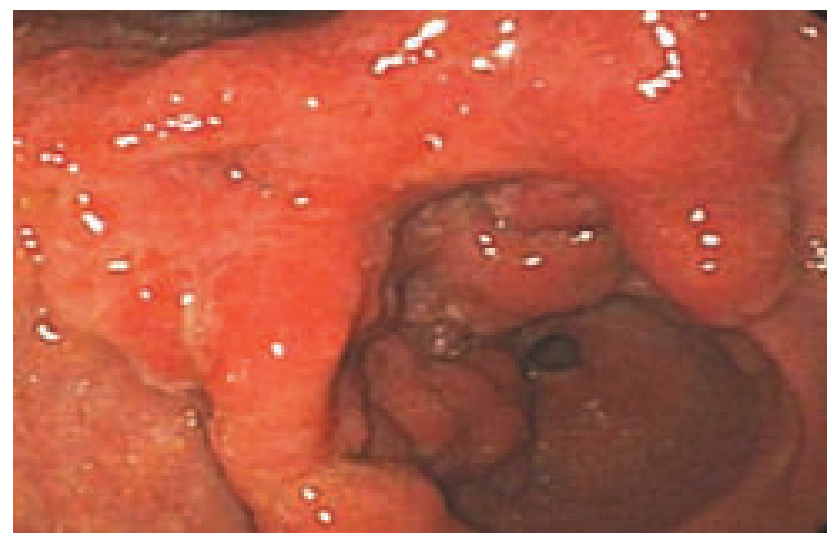

Figure 1: Nodular irregular growth at antrum of stomach

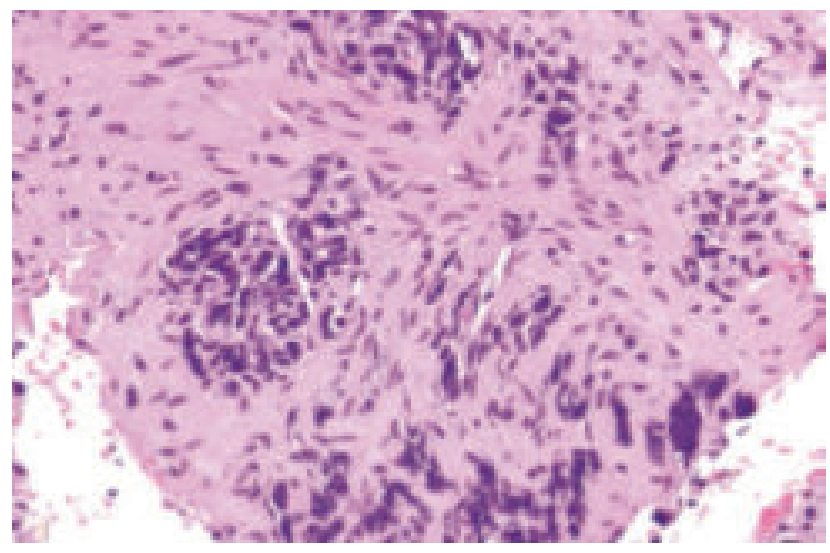

Figure 2: Poorly differentiated adenocarcinoma of stomach

\section{Discussion:}

Since its first description in 1962 MAHA was only published in few case reports. It can occur as a paraneoplastic syndrome in cancer patients, and may present as the first manifestation characterized by Coombs-negative hemolytic anemia with schistocytes and thrombocytopenia. The most common tumors associated with MAHA are gastric, breast, and lung cancers. ${ }^{3-5}$ A Korean study reported that 14 (25.5\%) out of 55 MAHA patients had gastric cancer. ${ }^{5}$ In a review of 168 reported cases of Cancer related-MAHA (CR- MAHA) by Lechner and Obermeier in 2012, the more frequently associated malignancies were adenocarcinomas of stomach, breast, prostate and lung. Another study included 60 patients with MAHA of different cancer origin revealed that approximately $50 \%$ of MAHA was associated to gastric carcinoma, followed by breast and lung cancer, $15 \%$ and $10 \%$ respectively. ${ }^{1} \mathrm{CR}-\mathrm{MAHA}$ is a rare and fatal complication of malignant tumors Most CR-MAHA patients die within a few weeks after the diagnosis, and the most common cause of death is infection. ${ }^{6}$ Our patient died within 4 weeks. There is only one case report in the literature where MAHA was associated to bone marrow carcinomatosis of a signet ring 
carcinoma of unknown origin. ${ }^{7}$ Most cases of MAHA have an abrupt onset. In our patient hemolytic anemia and thrombocytopenia and focal neurological deficit were the leading symptoms. These findings are in accordance with other cases and are typical for MAHA associated with TTP (thrombotic thrombocytopenic purpura).${ }^{8-10}$ Where systemic malignancy is not initially apparent, the combination of microangiopathic hemolytic anemia and thrombocytopenia in CR-MAHA is often misdiagnosed as TTP, a medical emergency. The classical pentad of TTP includes fever, microangiopathic hemolytic anemia, and thrombocytopenia, renal and neurological abnormalities. However, the urgency for its diagnosis in view of the effective early treatment options has decreased the stringency of these criteria. ${ }^{11}$ This exposes patients to the risks of plasma exchange therapy which is effective for TTP, but inappropriate in CR-MAHA. In fact, disseminated malignancy is an alternative disorder that only mimics TTP and is not TTP associated. ${ }^{12}$ Fatigue and dizziness mostly appear in advanced stages of anemia. Gastrointestinal bleedings can occur. A common and severe complication in advanced stages of disease are intracranial bleedings. ${ }^{13,14}$ One third of the patients present with jaundice due to hemolytic anemia, liver metastasis or extrahepatic bile duct obstruction. In this case the laboratory results may help getting the right diagnosis. Almost all patients present with hemolytic anemia, $50 \%$ of the patients present with hemoglobin levels $<8 \mathrm{~g} / \mathrm{dl}$. A characteristic finding in MAHA is the peripheral blood smear test with schistocytes which was also seen in our patients. Most patients present with platelet counts less than $50.000 / \mathrm{mm}^{3}$. Typical constellations which also appeared in our cases are a negative Coombs test, elevated lactic dehydrogenase levels. In patients with cancer associated MAHA renal failure is a rare condition but can appear in advanced stages. $1,15,16$

Today the pathogenesis of cancer associated MAHA is unclear. Whether tumor derived cell factors like platelet aggregating factors, antiendothelial cell antibodies or procoagulants are involved in MAHA is still under investigation. It is postulated that tumor cell emboli, together with immune complexes could generate endothelial damages which leads to platelet aggregation and endothelial proliferation. The characteristic fragmentation of red blood cells are due to the direct contact between red blood cells and the pathological arteries, arterioles and capillaries. These microvascular changes were reported in eight cases of cancer associated MAHA. ${ }^{14,}$ 17, 18

The prognosis of MAHA is extremely poor. Most patients die within a few weeks after diagnosis. ${ }^{1}$ Patients with bone marrow metastases and MAHA had significant worse median survival times compared to patients without MAHA (2 months versus 11 months, respectively). ${ }^{19}$ The bad outcome in patients with bone marrow metastases and MAHA needs special consideration. Bone marrow metastases occur in 1$11 \%$ of patients with gastric cancer. Examination of the histology has shown that more than $80 \%$ of bone metastasis from gastric cancer was poorly differentiated adenocarcinoma. Thoracic and lumbar vertebrae are the most frequent sites of bone metastasis. ${ }^{20}$ although there are reported cases of deposits in pelvis. ${ }^{21}$ Patients with bone metastases have mean survival times of less than 5 months with the longest survival period reported being 3 years. ${ }^{22}$ In one study median time leading to the diagnosis of bone marrow metastases was 3.5 years after curative gastrectomy (1 month - 5.8 years). Elevated alkaline phosphatase-, lactic dehydrogenase and c-reactive protein-levels may therefore help to identify patients with bone metastasis and MAHA.

\section{Conclusion:}

Till now no definitive treatment schedule exists for patients with MAHA. The acute onset of MAHA with hemolytic anemia and low platelet counts make a fast blood and platelet transfusion in nearly all patients necessary. An additional application of glucocorticoids is discussed controversial. ${ }^{14}$, ${ }^{23}$ The main focus of treatment is to reduce the tumor mass. A chemotherapy should be started as soon as possible after diagnosis. ${ }^{16}$ In our case showed, MAHA as the first side of metastatic disease, which is seen in approximately one third of all cases with MAHA. ${ }^{1}$ Therefore, laboratory findings which suggest MAHA make it necessary to search for the primary carcinoma.

\section{Conflict of interest: None}

\section{References:}

1. Antman KH, Skarin AT, Mayor RJ et al.: Microangiopathic hemolytic anemia and cancer: A review. Medicine 1979; 58:377-384

2. Ballas SK, Rubin RN: Case report: Microangiopathic hemolytic anemia and thrombocytopenia with disseminated cancer. Postgrad Med 1976;60:180-181

3. Elliott MA, Letendre L, Gastineau DA, Winters JL, Pruthi RK, Heit JA. Cancer-associated microangiopathic hemolytic anemia with thrombocytopenia: an important diagnostic consideration. Eur J Haematol 2010;85(1):43-50.

4. Kim YJ, Choi HM, Jung BG, Lee CK, Chung SY. Two cases of microangiopathic hemolytic anemia in patients with gastric cancer. Korean J Hematol 1990;25:575-9.

5. Youk DW, Choi MK, Kim KH, Kim HK, Park CW, Kim CC, Lee KS, Kim DJ, Kim BK. Microangiopathic hemolytic anemia in patients with gastrointestinal malignancy. Korean J Hematol 1983;18:207-14.

6. Arkenau HT, Müssig O, Buhr T, Jend HH, Porschen R. Microangiopathic hemolytic anemia (MAHA) as paraneoplastic syndrome in metastasized signet ring cell 
carcinomas: case reports and review of the literature. $\mathrm{Z}$ Gastroenterol 2005;43(8):719-22.

7. Nabeshima S, Kishihara Y, Nabeshima A et al.: Poorly differentiated adenocarcinoma with signet-ring cells of the Vater's ampulla, without jaundice but with disseminated carcinomatosis. Fukuoka Igaku Zasshi. 2003 Jul;94(7):23540.

8. Schiller D: Malignancy-associated microangiopathic hemolytic anemia.J Clin Oncol 1997; 2474-2475

9. Clinicopathologic Conference: Microangiopathic anemia and disseminated intravascular coagulation in an elderly woman. Am J Med 1983;74:1052-1060

10. Lynch EC, Bakken CL, Casey TH et al.: Microangiopathic hemolytic anemia in carcinoma of the stomach. Gastroenterology 1967;52:88-93

11. George JN. How I treat patients with thrombotic thrombocytopenic purpura: 2010. Blood 2010;116:4060-9. 10.1182/blood-2010-07-271445

12. Veyradier A, Meyer D. Thrombotic thrombocytopenic purpura and its diagnosis. J Thromb Haemost 2005;3:24207. $10.1111 / \mathrm{j} .1538-7836.2005 .01350$.

13. Goodnight SH: Bleeding and intravascular clotting in malignancy: A review. Ann NY Acad Sci 1974; 230:271-288

14. Joseph RR, Day HJ, Sherwin RM et al.: Microangiopathic hemolytic anemia associated with consumption coagulopathy in a patient with disseminated carcinoma. Scand J Haematol $1967 ; 4: 271-282$
15. Lohrmann HP, Adam W, Heymer B et al.: Microangiopathic hemolytic anemia in metastatic carcinoma: Report of eight cases. Ann Intern Med 1973;79:368-375

16. Marcos OO, Escuin E, Miguel JL et al.: Hemolytic syndrome in a patient with gastric adenocarcinoma: Partial recovery of renal function after gastrectomy. Clin Nephrol 1985;24:265268

17. Linklater Dm, Voth A: Thrombocytopenic purpura: Importance of early diagnosis. Can Fam Physician 1996; 42:1985-1991

18. Murgo AJ:Thrombotic microangiopathy in the cancer patient including those induced by chemotherapeutic agents. Seminars in Haematology 1987; 24:161-177

19. Tsuyoshi E, Hideo B, Akinobu T et al.: Diffuse bone metastasis with hematologic disorders from gastric cancer: Clinicopathological features and prognosis. Oncology reports 1999; 6:601-605

20. Carstens SA, Resnick D. Diffuse sclerotic skeletal metastasisas an initial feature of gastric carcinoma. Arch Intern Med1980;140(12):1666-8.

21. Chung YS, Choi TY, Ha CY, Kim HM, Lee KJ, Park CH, Fitzpatrick LA. An unusual case of osteoblastic metastasis from gastric carcinoma. Yonsei Med J2002;43(3):377-80.

22. Etoh T, Baba H, Taketomi A, Nakashima H, Kohnoe S, Seo Y, Fukuda T, Tomoda H. Diffuse bone metastasis with hematologic disorders from gastric cancer: clinicopathological features and prognosis. Oncol Rep 1999;6(3):601.

23. Miller SP, Davison T: Defibrination syndrome in cancer: Treatment with heparin. N Y Stae J Med 1967;67:452-458 